LUNG TRANSPLANTATION

\title{
Objective assessment of criteria for selection of donor lungs suitable for transplantation
}

\author{
A J Fisher, S C Donnelly, G Pritchard, J H Dark, P A Corris
}

Thorax 2004;59:434-437. doi: 10.1136/thx.2003.007542

See end of article for authors' affiliations ....................

Correspondence to: Professor P A Corris, Department of Thoracic Medicine, Freeman Hospital, High Heaton, Newcastle upon Tyne NE7 7DN, UK; paul.corris@ncl. ac.uk

Received 25 March 2003 Accepted

30 November 2003

\begin{abstract}
Background: Donor organ shortage severely limits lung transplantation as a therapeutic option, yet many potential donor lungs are deemed unsuitable by clinical selection criteria.

Methods: Of 39 consecutive potential donor lungs, 14 were accepted and 25 excluded by clinical selection criteria. All were evaluated prospectively by clinical assessment, bronchoscopy, and bronchoalveolar lavage (BAL) to evaluate objectively the discrimination of pulmonary infection and injury. Results: Accepted donors were significantly younger than those excluded (mean (SD) age 36.7 (15.3) years $v 49.5$ (13.2) years; $p=0.009$, unpaired $t$ test) and were more likely to have suffered traumatic brain death (50\% v 20\%; $\mathrm{p}=0.07$, Fisher's exact test). Oxygenation $\left(\mathrm{PaO}_{2}: \mathrm{FiO}_{2}\right)$ was higher in accepted donors than in excluded donors (median (range) 63.2 (48-82.5) kPa $\vee 43.1$ (7.7-71.7) kPa; $p=0.0001$, Mann-Whitney test). Positive formal BAL culture was more frequent in accepted donors (75\%) than in those excluded (43\%; $p=0.1$, Fisher's exact test). There was no significant difference in the percentage and concentration of neutrophils in BAL fluid between accepted and excluded donors (median (range) $37.9(0-96.9) \%$ and $44.6(0-1190) \times 10^{3} / \mathrm{ml} v 36(1-98.1) \%$ and $\left.46(0.2-1457) \times 10^{3} / \mathrm{ml}\right)$, nor in the BAL fluid concentration of tumour necrosis factor- $\alpha(140(0-340) \mathrm{pg} / \mathrm{ml} v 160(0-760) \mathrm{pg} / \mathrm{ml})$ or interleukin 8 (810 (33-17 600) pg/ml v $540(0-15110) \mathrm{pg} / \mathrm{ml})$.

Conclusion: Current selection criteria are poor discriminators of pulmonary injury and infection and lead to the exclusion of potentially usable donor lungs.
\end{abstract}

ung transplantation is now accepted as a treatment option which offers hope of long term survival in selected - patients with end stage respiratory disease. ${ }^{1}$ The increasing expertise worldwide in both surgical technique and in the management of post-transplant complications has led to a dramatic improvement in the success of the procedure. The major hurdle to the more widespread application of lung transplantation is the severe shortage of donor organs. Concerted efforts to improve rates of organ donation via publicity campaigns have failed to produce a dramatic increase, suggesting that other strategies are now required to increase the number of lung transplantations performed annually in the UK. The lack of donor organs both limits the eligibility of patients to be placed on the waiting list and contributes dramatically to the mortality of those already on the waiting list, currently $30-40 \%{ }^{2}$

Statistics compiled by the UK Transplant Services Support Authority (UKTSSA) on all multi-organ donors within the UK reveal that many potential donor lungs are deemed unsuitable and excluded from use by clinical selection criteria. ${ }^{3}$ Currently, less than $25 \%$ of all brain dead donors are deemed suitable for use in clinical lung transplantation. A large number of donor lungs are therefore never used, despite consent being given by the next of kin.

The current selection criteria, used as a guide, were drawn up over 20 years ago by expert consensus and have changed little since (box 1). There is, however, no evidence base to support them as they are based on proposed best practice. In many centres those lungs which do not completely satisfy the criteria can be used at the discretion of the duty transplant surgeon. ${ }^{4}$ This obviously leads to differences between and within centres in interpretation of organ suitability.

Organ donors arise from a population of severely brain injured patients. The use of only a small proportion of the donor lungs offered reflects the fact that the lungs are very susceptible to compromise in brain dead patients. ${ }^{5}$ An approach aiming to increase the use of donor lungs which do not completely satisfy the criteria has been postulated as a possible way of increasing the donor lung pool. ${ }^{6}$ Acceptance of only $25 \%$ of those currently excluded would double the number of lung transplants that could be performed and significantly reduce mortality on the waiting list. ${ }^{7}$ Several studies have retrospectively assessed the outcome and early graft function in recipients of non-ideal or "marginal" donor lungs and have shown no significant difference in early graft function between the two groups. ${ }^{8-10}$ This suggests that a large number of potential donor lungs which are currently excluded may have been successfully used in lung transplantation.

There is a scarcity of any published data which has prospectively investigated current donor selection criteria using objective measures. The use of objective measures of donor lung viability, degree of injury, and potential function would allow an appropriate maximal use of this scarce resource. We therefore aimed, in a prospective manner, to determine how effective the current method of donor lung selection is at identifying lung injury as measured by objective indices of infection and inflammation.

\section{METHODS}

Ventilated patients identified by regional intensive care unit (ICU) staff as potential organ donors were referred to our zonal donor coordinator for further assessment. Those who fulfilled the criteria for the diagnosis of brainstem death and whose relatives had given consent for organ donation and research procedures were prospectively included in the study over an 18 month recruitment period (1998-9). Approval for the study was granted by the regional ethics committee.

\section{Clinical methods}

All patients underwent a detailed clinical evaluation as part of their assessment to be potential organ donors. Using this 
Box 1 Criteria for selection of suitable lung donors (adapted from Winton ${ }^{4}$ )

- Age $<65$ years

- No history of severe active lung disease

- Limited smoking history

- No history of malignant neoplasm

- HIV and hepatitis B and C negative

- No evidence of active pulmonary sepsis

- Clear chest radiograph

- Insignificant endotracheal secretions

- $\mathrm{PaO}_{2}>60 \mathrm{kPa}$ on $\mathrm{FiO}_{2} 1.0$

- Acceptable visual inspection of lungs

- Acceptable bronchoscopic findings

information in conjunction with accepted selection criteria, a decision on whether to accept or exclude the lungs from use in transplantation was made by the duty cardiopulmonary transplant surgeon in line with standard clinical practice and without knowledge of objective measures.

The reason for exclusion was noted and all patients were then separated into those deemed suitable lung donors and those excluded by interpretation of current selection criteria.

Fibreoptic bronchoscopy and bronchoalveolar lavage (BAL) was performed on all the potential donors in the operating theatre of the donor hospital by the same investigator. The visual appearance of the endobronchial tree was noted. BAL was performed by standard techniques. Briefly, two $60 \mathrm{ml}$ aliquots of sterile saline were instilled into the right middle lobe or lingula and aspirated by gentle hand suction to avoid cell damage. The lavage fluid was stored on ice until return to the base laboratory. Ten $\mathrm{ml}$ of lavage fluid was used for formal microbiological evaluation to detect bacteria, viruses, and fungi.

\section{Laboratory methods}

The lavage fluid was filtered through gauze to remove mucoid debris and centrifuged at $1000 \mathrm{~g}$ for $4 \mathrm{~min}$ at $4^{\circ} \mathrm{C}$ to separate a cell pellet from the supernatant. The supernatant was further centrifuged at $2500 \mathrm{~g}$ for $4 \mathrm{~min}$ at $4^{\circ} \mathrm{C}$ and aliquoted into small volumes before storage at $-70^{\circ} \mathrm{C}$ for subsequent analysis. Total cell numbers were determined in the resuspended pellet using a haemocytometer. Cytospins of the cell suspension were then prepared (Shandon 3 cytospin) and stained with a modified Giemsa stain on an automated staining console (Haematec). The differential cell count was determined from the high power field examination of 1000 cells.

Concentrations of the acute proinflammatory cytokines tumour necrosis factor $\alpha$ (TNF- $\alpha$ ) and interleukin 8 (IL-8) in BAL fluid were determined by a previously established ELISA technique. ${ }^{11}$ Briefly, mouse antibody to human IL-8 (R\&D Systems, UK) was bound to microtitre plates (Costar, Cambridge, MA, USA) by overnight incubation. Endogenous binding was blocked with $0.1 \%$ bovine serum albumin in phosphate buffered saline (PBS). Plates were washed three times between each step with PBS containing $0.05 \%$ Tween 20. Human IL-8 (R\&D Systems, UK) in duplicate serial dilutions $10 \mathrm{pg} / \mathrm{ml}$ to $100 \mathrm{ng} / \mathrm{ml}$ was used to generate a standard curve. Samples in triplicate $(100 \mu \mathrm{l}$ of neat lavage) were incubated in wells for 2 hours at room temperature before adding biotin labelled anti-human IL-8 detection antibody (R\&D Systems, UK) for a further 2 hours. Each well was incubated for $30 \mathrm{~min}$ with streptavidin- peroxidase conjugate before chromogen (tetramethylbenzidine, Sigma) was added and developed over 10-15 min in darkness. The reaction was terminated using $50 \mu \mathrm{l} \mathrm{lM}$ sulphuric acid and optical absorption measured at $450 \mathrm{~nm}$ (Dynatec, MR5000). IL-8 concentrations were quantified against the linear portion of the standard curve using analysis software (Assayzap). To establish whether the levels of other powerful neutrophil chemokines in the donor BAL fluid affected outcome in the recipient, the quantities of TNF- $\alpha$ were determined using similar methodologies.

\section{Statistical methods}

The potential donors were divided into two groups based on the decision to accept or exclude them from use in clinical lung transplantation. Differences between the two groups with respect to numerical indices were assessed where possible using parametric analysis (unpaired Student's $t$ test). When the numerical data were non-normally distributed, the differences between the two groups were determined by non-parametric methods (Mann-Whitney U test). Categorical data such as the cause of brain death were compared between the two groups using the Fisher exact test.

\section{RESULTS}

In the UK northern organ retrieval zone, over an 18 month period 39 ventilated patients who fulfilled the criteria for brainstem death and whose relatives had consented to organ donation were included in the study.

Fourteen potential donors $(35.9 \%)$ satisfied the clinical selection criteria and were accepted by the duty transplant surgeon as being suitable for use as lung donors in clinical lung transplantation. The remaining 25 (64.1\%) failed to satisfy the selection criteria and were deemed unsuitable. The accepted donors provided lungs for five bilateral sequential lung transplants, seven single lung transplants, and two heart-lung transplants. Fibreoptic bronchoscopy and standard BAL was attempted in all 39 donors. However, in two the procedure was abandoned due to cardiovascular instability in the donor. Of the 37 donor lavages completed, four failed to yield the minimum instilled volume return of $30 \%$ and were of unsuitable quality for cellular and molecular assessment but were sent for formal microbiological culture. Cellular and molecular assessment of BAL fluid was therefore performed on 12 donors accepted for use and on 21 excluded donors.

The demographic comparisons between those donors accepted for use and those excluded are shown in table 1. Accepted donors were significantly younger than those excluded as suitable donors (mean (SD) 36.7 (15.3) years $v$ 49.5 ( 13.2 ) years; $\mathrm{p}=0.009$, unpaired $t$ test).

Fifty percent of the accepted donors suffered brain death as a result of trauma such as a road traffic accident or head injury, compared with only $20 \%$ of the excluded donors who were more likely to have suffered a spontaneous intracranial event such as stroke or subarachnoid haemorrhage $(p=0.07$, Fisher's exact test). There was no significant difference in the duration of mechanical ventilation before donor assessment or in current smoking history between accepted and excluded donors.

The most common reason for excluding a potential donor was poor gas exchange indicated by a low $\mathrm{PaO}_{2}: \mathrm{FiO}_{2}$ ratio $(n=7)$. Clinical suspicion of infection or aspiration accounted for five and four, respectively. Five subjects were excluded because of an unfavourable appearance on the chest radiograph, two because of an abnormality on visual inspection (large bullae in one and lung contusion in another), and two were excluded for other reasons (age 69 years and lack of a suitable recipient who could be transferred to the transplant centre in sufficient time). 
Table 1 Demographic and clinical indices of donors accepted and excluded from use in lung transplantation

\begin{tabular}{|c|c|c|c|c|c|}
\hline Donor outcome & Mean (SD) age (years) & Trauma, n (\%) & Current smoker, n (\%) & Mean (SD) hours of ventilation & Median (range) $\mathrm{PaO}_{2}: \mathrm{FiO}_{2}(\mathrm{kPa})$ \\
\hline Accepted $(n=14)$ & $36.7(15.3)$ & $7(50 \%)$ & $4(28 \%)$ & $45.6(16.8)$ & $63.2(48-82.5)$ \\
\hline Excluded $(n=25)$ & $49.5(13.2)$ & $5(20 \%)$ & $12(48 \%)$ & 35.5 (14.4) & $43.1(7.7-71.7)$ \\
\hline$p$ value & $0.009^{*}$ & $0.07 \dagger$ & $0.32 \dagger$ & $0.057^{*}$ & $0.0001 \ddagger$ \\
\hline
\end{tabular}

\section{Clinical evaluation of donors}

The oxygenation capacity of the potential donor lungs as measured by $\mathrm{PaO}_{2}: \mathrm{FiO}_{2}$ was significantly higher in accepted donors than in those excluded (median (range) 63.2 (4882.5) $\mathrm{kPa} v 43.1$ (7.7-71.7) $\mathrm{kPa} ; \mathrm{p}=0.0001$, Mann-Whitney $\mathrm{U}$ test). The clinical suspicion of significant pulmonary sepsis, as suggested by suctioning of purulent secretions from the endotracheal tube, correlated poorly with visual inspection of the central airways at bronchoscopy. Also, the pre-assessment report of possible aspiration correlated poorly with that witnessed by bronchoscopy. The major airways of three of the four donors excluded for possible aspiration were found to be clear by bronchoscopy, and three of the 14 accepted donors had evidence of thick purulent lower airway secretions, significant bronchial wall reddening, and oedema despite a clear chest radiograph.

Chest radiographic evaluation performed within 24 hours of donor assessment was clear in nine $(36 \%)$ of the 25 excluded donors and seven (50\%) of the accepted donors. Most of the abnormal radiographs had bilateral shadowing either in the bases or more diffusely.

\section{Laboratory evaluation of donors}

There was a high incidence of infection in the lungs of the donor population, with $18(48.6 \%)$ potential donors having positive cultures of lavage fluid. The following species were identified: Staphylococcus aureus $(\mathrm{n}=7)$, coliform species $(\mathrm{n}=4)$, Candida albicans $(\mathrm{n}=3)$, Haemophilus influenzae $(\mathrm{n}=3)$, Streptococcus pneumoniae $(\mathrm{n}=1)$, Enterobacter species $(\mathrm{n}=1)$. The incidence of positive cultures tended to be higher in the BAL fluid of subjects accepted as suitable lung donors $(75 \%)$ than in those excluded $(43 \%)$, although the difference did not achieve statistical significance $(p=0.1$, Fisher's exact test). Differences in the markers of inflammatory activity in the BAL fluid of accepted and excluded donors are shown in table 2. No significant differences were seen in the percentage and concentration of neutrophils or in the concentrations of TNF- $\alpha$ or IL- 8 in the BAL fluid of accepted and excluded lung donors.

\section{DISCUSSION}

The shortage of donor organs is a major problem for all solid organ transplantation programmes. This shortage, however, is felt most acutely in lung transplantation where the susceptibility of the donor lung to dysfunction and the desire to achieve the best outcome means only a small proportion of potential donor lungs are deemed suitable for implantation into a recipient. This problem has led to consideration of possible alternatives to cadaveric lung donation including xenotransplantation, non-heart beating donors (post-cardiac arrest), and living donor lobar transplantation. ${ }^{12}$ Only the last of these alternatives is currently available, and although there is much ongoing research, it offers no solution to the problem in the foreseeable future. Living donor lobar transplantation is being used in highly selected recipients where it can be successful, but the technique is not suitable for the majority of patients on the lung transplant waiting list. ${ }^{13}$ The need to re-evaluate the way donor organs are selected and, in particular, the reasons why more than $75 \%$ of donor lungs are excluded has previously been suggested as a more appropriate and achievable way of increasing the supply of donor organs. ${ }^{7}$ The current selection criteria are based on good clinical judgement and are historical in origin, but have no evidence base.

In this study we have evaluated, in a group of human cadaveric donors, the subjective decision to exclude donors from use in transplantation based on clinical assessment and compared this with an objective assessment of the donor lung with cellular and molecular measures of infection and injury.

The major clinical difference between the two groups was in gas exchange as measured by $\mathrm{PaO}_{2}: \mathrm{FiO}_{2}$. This is not surprising as the oxygenation capacity of the potential lung provides one of the primary selection criteria. Only seven of the 25 donors excluded, however, had a low $\mathrm{PaO}_{2}: \mathrm{FiO}_{2}$ as the primary reason for exclusion. There is no evidence from clinical lung transplantation to support the concept that the function of the donor lung after implantation is related to the donor's $\mathrm{PaO}_{2}: \mathrm{FiO}_{2}$. In fact, studies previously conducted on marginal donors have suggested that comparable immediate graft function can be achieved with use of donors with $\mathrm{PaO}_{2}: \mathrm{FiO}_{2}$ below that recommended for selection. ${ }^{10}{ }^{14}$ This suggests that donor lungs may function very differently when removed from the critical illness environment. Furthermore, a recent report has demonstrated the importance of quantifying the contribution to gas exchange made by each lung segment. Pulmonary vein gas analysis may reveal that one lung is functioning much more effectively than the other and allow its use for single lung transplantation. ${ }^{15}$ We believe that donors with $\mathrm{a} \mathrm{PaO}_{2}$ of $\geqslant 30 \mathrm{kPa}$ on $100 \%$ oxygen should be carefully considered for use with differential pulmonary vein gas assessment, the better side being used as a single donor lung. Alternatively, the pair of lungs could be used for a bilateral lung transplant in a relatively fit recipient who could tolerate a degree of early graft dysfunction.

The chest radiograph may give some clues to the cause of poor gas exchange and may help to localise an abnormality to one hemithorax, allowing use of the unaffected lung. However, many of the abnormalities seen were bilateral

Table 2 Inflammatory and infective indices of donors accepted and excluded from use in lung transplantation

\begin{tabular}{llllll}
\hline Donor outcome & Neutrophils $(\%)$ & Neutrophils $\left(\times 10^{3} / \mathrm{ml}\right)$ & IL-8 $(\mathrm{pg} / \mathrm{ml})$ & TNF- $\alpha(\mathrm{pg} / \mathrm{ml})$ & No $(\%)$ culture positive \\
\hline Accepted $(n=12)$ & $37.9(0-96.9)$ & $44(0-1200)$ & $810(0-17600)$ & $140(0-340)$ & $9(75 \%)$ \\
Excluded $(n=21)$ & $36.0(0-98.1)$ & $46(0-1400)$ & $540(0-15100)$ & $160(0-76)$ & $9(43 \%)$ \\
p value & $0.49 \dagger$ & $0.78 \dagger$ & $0.64 \dagger$ & $0.63 \dagger$ & $0.1 \neq$ \\
\hline
\end{tabular}

*Median (range).

†Mann-Whitney $U$ test.

fFisher's exact test. 
and diffuse, representing a well recognised clinical phenomenon-the pathophysiology of which is not fully understood-often labelled neurogenic pulmonary oedema. There is growing evidence that acute lung injury can result from brain death itself. ${ }^{16}$ We believe that localised unilateral changes on the radiograph should not preclude use of the contralateral lung for single lung transplantation or both lungs for bilateral transplantation in an otherwise fit recipient.

Infection is a major cause of early death following lung transplantation and screening of the donor lung for sepsis is therefore vital. The presence of purulent secretions alone suctioned from the endotracheal tube is often sufficient to lead to donor lung exclusion, especially if supported by positive Gram staining. However, a recent study has suggested that Gram staining of endobronchial secretions is a poor predictor of outcome after lung transplantation and should not be used in the selection criteria. ${ }^{17}$ Our results have shown a poor correlation between Gram staining and formal culture of lavage, suggesting that clinical suspicion is inadequate for identifying infection in the lower respiratory tract. We believe that positive Gram staining on tracheal secretions should not exclude use of the organs if the lower airways appear clear on bronchoscopic examination. Alternative approaches to identifying lower airway infection such as culture of protected brush specimens may prove invaluable, not necessarily in the selection of donors but in directing antimicrobial treatment after transplantation.

No differences were seen between the two groups of donors in the cellular indices used as objective markers of infection and inflammation, suggesting that the degree of inflammatory activation is similar. We have previously shown that the degree of inflammation in the donor lung before implantation affects early outcome in the recipient. ${ }^{18}$ In particular, raised IL-8 levels in donor lavage fluid were associated with a risk of early graft dysfunction and early mortality. There was also a suggestion that the degree of intra-alveolar neutrophilia transferred to the recipient with the donor lung may be important, but this did not achieve statistical significance. ${ }^{18}$ In our current evaluation the numbers of neutrophils and the levels of IL- 8 are highly comparable between the two groups. The use of these novel markers of lung inflammation and injury offer a potential new approach to donor lung assessment but require more research before being used in clinical practice.

The findings of this study support the hypothesis that the current selection criteria may lead to the exclusion of potentially usable donor lungs. Our findings support those of a recently published study by Ware and colleagues ${ }^{19}$ which examined the physiological function of donor lungs excluded from transplantation. By assessing alveolar fluid clearance and extravascular water to dry weight ratios, the authors claim that over $40 \%$ of the donor lungs could have been successfully used. This study, however, has limitations as they found that the physiological measurements were a surrogate for the $\mathrm{PaO}_{2}$ in the donor, which is already part of the assessment criteria. Furthermore, no prospective evidence exists to suggest that better alveolar fluid clearance in donors predicts better graft function after implantation.

However, our findings support the view of Ware and colleagues that increasing the share of the cadaveric donor population accepted for use could dramatically increase the supply of organs for lung transplantation. As a result of our findings we have in Newcastle, as have other large centres, significantly relaxed our donor selection criteria and increased our acceptance rate. It is important that initially we attempt to understand more fully the pathophysiology of lung injury after brain death and then devise ways of protecting the donor lungs from injury. We share the opinion of Weill and colleagues from the University of Alabama that a large multicentre database of donor characteristics and early recipient outcomes would aid the identification of more evidence based indicators of potential graft function after implantation. ${ }^{20}$ We believe that the International Society for Heart and Lung Transplantation (ISHLT) should take a lead in updating donor lung selection criteria to a new international standard. This, together with the database previously described, would enable donor lung use to be maximised and provide more accurate risk stratification for any potential lung transplant recipient.

\section{Authors' affiliations}

A J Fisher, G Pritchard, J H Dark, P A Corris, Department of

Cardiopulmonary Transplantation and Immunobiology and

Transplantation Research Group, University of Newcastle upon Tyne,

Freeman Hospital, High Heaton, Newcastle upon Tyne NE7 7DN, UK

S C Donnelly, Department of Medicine and Therapeutics, St Vincent's

Hospital and Conway Institute, University College Dublin, Dublin, Ireland

\section{REFERENCES}

1 Trulock EP. Lung transplantation. Am J Respir Crit Care Med 1997; 155:789-818.

2 Anyanwu AC, Rogers CA, Murday AJ, et al. Intrathoracic organ transplantation in the United Kingdom 1995-99: results from the UK cardiothoracic transplant audit. Heart 2002;87:449-54.

3 United Kingdom Transplant Services Support Authority (UKTSSA) Cardiothoracic organ transplant audit 1985-1995. Bristol: UKTSSA, 1996

4 Winton TL. Lung transplantation: donor selection. Semin Thorac Cardiovasc Surg 1992;4:79-82.

5 Fisher AJ, Donnelly SC, Hirani N, et al. Enhanced pulmonary inflammation in organ donors following fatal non-traumatic brain injury. Lancet 1999;353:1412-3.

6 Shumway SJ, Hertz MI, Petty MG, et al. Liberalization of donor criteria in lung and heart-lung transplantation. Ann Thorac Surg 1994;57:92-5.

7 Fisher AJ, Dark JH, Corris PA. Improving donor lung evaluation-a new approach to increase organ supply for lung transplantation. Thorax 1998;53:818-20.

8 Bhorade SM, Vigneswaran W, McCabe MA, et al. Liberalization of donor criteria may expand the donor pool without adverse consequence in lung transplantation. J Heart Lung Transplant 2000;19:1199-204.

9 Kron IL, Tribble CG, Kern JA, et al. Successful transplantation of marginally acceptable thoracic organs. Ann Surg 1993;217:518-22.

10 Sundaresan S, Semenkovich J, Ochoa L, et al. Successful outcome of lung transplantation is not compromised by the use of marginal donor lungs. J Thorac Cardiovasc Surg 1995;109:1075-9.

11 Donnelly SC, Strieter RM, Kunkel SL, et al. Interleukin-8 and development of adult respiratory distress syndrome in at-risk patient groups. Lancet 1993;341:643-7.

12 Grover FL, Fullerton DA, Zamora MR, et al. The past, present, and future of lung transplantation. Am J Surg 1997; 173:523-33.

13 Starnes VA, Woo MS, Maclaughlin EF, et al. Comparison of outcomes between living donor and cadaveric lung transplantation in children. Ann Thorac Surg 1999;68:2279-83.

14 Pierre AF, Sekine Y, Hutcheon MA, et al. Marginal donor lungs: a reassessment. J Thorac Cardiovasc Surg 2002;123:421-7.

15 Aziz TM, El-Gamel A, Saad RA, et al. Pulmonary vein gas analysis for assessing donor lung function. Ann Thorac Surg 2001;73:1599-604.

16 Takada M, Nadeau KC, Hancock WW, et al. Effects of explosive brain death on cytokine activation of peripheral organs in the rat. Transplantation 1998;65:1533-42.

17 Weill D, Dey G, Hick R, Young K, et al. A positive donor gram stain does not predict outcome following lung transplantation. J Heart Lung Transplant 2002;21:555-8

18 Fisher AJ, Donnelly SC, Hirani N, et al. Elevated levels of interleukin-8 in donor lungs is associated with early graft failure after lung transplantation. Am J Respir Crit Care Med 2001;163:259-65.

19 Ware L, Wang Y, Fang X, et al. Assessment of lungs rejected for transplantation and implications for donor selection. Lancet 2002;360:619-20.

20 Weill D. Donor criteria in lung transplantation-an issue revisited. Chest 2002;121:2029-31. 